\title{
UNIQUENESS AND THE CONVERGENCE OF SUCCESSIVE APPROXIMATIONS
}

\author{
A. D. ZIEBUR
}

Picard's method of solving the differential problem

$$
x^{\prime}=f(t, x) \text { for } t>t_{0}, \quad x=x_{0} \text { when } t=t_{0}
$$

consists of finding the limit of a sequence $\left\{\xi_{n}(t)\right\}$ that is defined as follows. An initial function $\xi_{0}$ is chosen, and the remaining members of the sequence are generated by the recursion formula

$$
\xi_{n+1}(t)=x_{0}+\int_{t_{0}}^{t} f\left(s, \xi_{n}(s)\right) d s .
$$

If the function $f$ is continuous, and if it satisfies a Lipschitz condition in its second argument, then the convergence (in a sufficiently small interval) of the sequence $\left\{\xi_{n}(t)\right\}$ is assured. In addition, the uniqueness of the solution of (1) is guaranteed. This association of the uniqueness of the solution of (1) and the convergence of the Picard sequence $\left\{\xi_{n}(t)\right\}$ has spurred considerable study into the relationship between these two phenomena. For example, conditions have been found which are weaker than the Lipschitz condition but which still guarantee uniqueness and convergence. There are, however, examples that show that uniqueness and convergence do not go hand in hand. Amplification of these statements, along with a short bibliography of the problem, may be found in Chapter 2 of the book of Coddington and Levinson [2]. Mention should also be made of the paper of LaSalle [3] and the references listed there. In the present note we will show that, although the uniqueness of the solution of (1) does not in general imply the convergence of the Picard sequence $\left\{\xi_{n}(t)\right\}$, there is a differential problem of the second order associated with (1), the uniqueness of whose solution does guarantee the convergence of $\left\{\xi_{n}(t)\right\}$. It will be seen that the arguments we present produce results which are valid "in the small"; these results may be continued by the standard techniques used in the study of differential equations.

We will assume that our function $f$ is continuous and is of "bounded variation in its second argument." More specifically, we suppose that we can write $f(t, x)=I(t, x)+D(t, x)$, where $I$ and $D$ are continuous in a domain that contains the point $\left(t_{0}, x_{0}\right)$ and are, respectively, "increasing" and "decreasing" in their second argument, in the sense that if $u>v$, then $I(t, u) \geqq I(t, v)$, while $D(t, u) \leqq D(t, v)$. Now we

Received by the editors January 9, 1962. 
consider the differential problem of the second order

$$
\begin{gathered}
y^{\prime}=I(t, y)+D(t, z), \quad z^{\prime}=I(t, z)+D(t, y) \\
\text { for } t>t_{0},(y, z)=\left(x_{0}, x_{0}\right) \text { when } t=t_{0} .
\end{gathered}
$$

It is clear that if $x$ is a solution of (1), then the pair $(x, x)$ is a solution of (3). Conversely, of course, if $(y, z)$ is a solution of (3), and if $y=z$, then this common value is a solution, say $x$, of (1). We will see below that the condition that the solution of (3) be unique is that all the solutions of (3) are of the form $(y, z)=(x, x)$, that is, if $(y, z)$ is a solution, then $y=z$. It turns out that the uniqueness of the solution of (3) guarantees both the uniqueness of the solution of (1) and the convergence of the Picard sequence $\left\{\xi_{n}(t)\right\}$ to this solution.

The main tool that we use to prove this assertion is the following lemma.

LEMmA. If $\omega$ and $\Omega$ are two functions such that

$$
\begin{aligned}
\Omega^{\prime}(t) \leqq I(t, \Omega(t))+D(t, \omega(t)), & \omega^{\prime}(t) \geqq I(t, \omega(t))+D(t, \Omega(t)), \\
\omega(t) \leqq \Omega(t) \quad \text { for } t>t_{0}, & \omega\left(t_{0}\right)=\Omega\left(t_{0}\right)=x_{0},
\end{aligned}
$$

and if all the solutions of (3) have the form $(x, x)$, then $\omega(t)=\Omega(t)$ for $t>t_{0}$.

Proof. Let $\epsilon$ be a positive number and suppose that $\left(\eta_{\epsilon}(t), \zeta_{\epsilon}(t)\right)$ is a solution of the problem

$$
\begin{aligned}
y^{\prime} & =I(t, y)+D(t, z)+\epsilon, \quad z^{\prime}=I(t, z)+D(t, y)-\epsilon \text { for } t>t_{0}, \\
(y, z) & =\left(x_{0}, x_{0}\right) \quad \text { when } t=t_{0} .
\end{aligned}
$$

We will show that

$$
\zeta_{\epsilon}(t) \leqq \omega(t) \leqq \Omega(t) \leqq \eta_{\epsilon}(t) .
$$

As $\epsilon \rightarrow 0,\left(\eta_{\epsilon}(t), \zeta_{\epsilon}(t)\right)$ approaches a solution of (3). By hypothesis, all solutions of (3) have the form $(x, x)$, and so $\lim _{\epsilon \rightarrow 0} \eta_{\epsilon}(t)=\lim _{\epsilon \rightarrow 0} \zeta_{\epsilon}(t)$. Then it follows from (6) that $\omega(t)=\Omega(t)$. Thus to prove our lemma we must show that (6) holds for each point $t$ to the right of $t_{0}$, and so in the remainder of this proof we assume that $t$ is some definite point to the right of $t_{0}$. Suppose we let $\left[t_{0}, u\right]$ be the maximum closed subinterval of $\left[t_{0}, t\right]$ that contains $t_{0}$ and is such that $\Omega(s) \leqq \eta_{\epsilon}(s)$ for each point $s$ in $\left[t_{0}, u\right]$ and let $\left[t_{0}, v\right]$ be the maximum closed subinterval that contains $t_{0}$ and is such that $\zeta_{\epsilon}(s) \leqq \omega(s)$ for each point $s$ in $\left[t_{0}, v\right]$. In defining these subintervals we do not exclude the possibility that one or both may be the degenerate interval $\left[t_{0}, t_{0}\right]$, but now we will show that actually $u=v=t$, which will verify (6). We may do this 
by showing that each of the suppositions $u=v<t, u<v \leqq t$, and $v<u \leqq t$ leads to a contradiction. We first observe that because of the continuity of our functions, and because of our initial condition, $\Omega(u)=\eta_{\epsilon}(u)$ if $u<t$ and $\omega(v)=\zeta_{\epsilon}(v)$ if $v<t$. Therefore if $u=v<t$ there is a neighborhood $[u, r]$, where $u<r \leqq t$, such that for $s$ in $[u, r]$,

$$
I(s, \Omega(s))+D(s, \omega(s)) \leqq I\left(s, \eta_{\epsilon}(s)\right)+D\left(s, \zeta_{\epsilon}(s)\right)+\epsilon .
$$

Because $\left(\eta_{\epsilon}(t), \zeta_{\epsilon}(t)\right)$ is a solution of $(5)$, the right side of (7) equals $\eta_{\epsilon}^{\prime}(s)$, and by (4) the left side is not less than $\Omega^{\prime}(s)$. Thus $\Omega^{\prime}(s)$ $\leqq \eta_{\epsilon}^{\prime}(s)$, which implies, since $\Omega(u)=\eta_{\epsilon}(u)$, that $\Omega(s) \leqq \eta_{\epsilon}(s)$ for each $s$ in $[u, r]$. But then we see that $\Omega(s) \leqq \eta_{\epsilon}(s)$ for each $s$ in $\left[t_{0}, r\right]$, which contradicts the maximality of $\left[t_{0}, u\right]$. If we assume that $u<v \leqq t$, we can arrive at this same contradiction. For we have $\Omega(u)=\eta_{\epsilon}(u)$ and, since $u$ belongs to the interval $\left[t_{0}, v\right], \zeta_{\epsilon}(u) \leqq \omega(u)$. It follows (we use the fact that $D$ is decreasing in its second argument) that $I(u, \Omega(u))$ $+D(u, \omega(u))<I\left(u, \eta_{\epsilon}(u)\right)+D\left(u, \zeta_{\epsilon}(u)\right)+\epsilon$, and hence again there is an interval $[u, r]$ in which (7) holds. Now our argument proceeds as before. We rule out the possibility that $v<u \leqq t$ in a similar manner.

From this lemma we see that if all the solutions of (3) have the form $(x, x)$, then the solution of $(1)$ is unique. For suppose that $\omega$ and $\Omega$ are the minimum and maximum solutions of (1). Then $\omega^{\prime}(t)$ $=I(t, \omega(t))+D(t, \omega(t))$ and $\Omega^{\prime}(t)=I(t, \Omega(t))+D(t, \Omega(t))$. Because $D$ is decreasing in its second argument, we neither increase the right side of the first of these equations nor decrease the right side of the second if we interchange $D(t, \omega(t))$ and $D(t, \Omega(t))$. Therefore this interchange gives us (4), and hence our lemma tells us that $\omega(t)=\Omega(t)$. At this point it is clear that the statement "all the solutions of (3) have the form $(x, x)$ " is equivalent to the statement "(3) has a unique solution."

To show how the uniqueness of the solution of (3) implies the convergence of the Picard sequence $\left\{\xi_{n}(t)\right\}$, let us write

$$
\Lambda(t)=\lim \sup \xi_{n}(t)=x_{0}+\lim \sup \int_{t_{0}}^{t} f\left(s, \xi_{n}(s)\right) d s
$$

and

$$
\lambda(t)=\lim \inf \xi_{n}(t)=x_{0}+\liminf \int_{t_{0}}^{t} f\left(s, \xi_{n}(s)\right) d s .
$$

Fatou's Lemma [2, p. 194] says that we do not increase this last expression if we take the symbol lim inf inside the integral sign. Thus $\lambda(t) \geqq x_{0}+\int_{t_{0}}^{t} \lim \inf f\left(s, \xi_{n}(s)\right) d s$. Now $\lim \inf f\left(s, \xi_{n}(s)\right)$ $\geqq \lim \inf I\left(s, \xi_{n}(s)\right)+\lim \inf D\left(s, \xi_{n}(s)\right)=I(s, \lambda(s))+D(s, \Lambda(s))$. There- 
fore $\lambda(t) \geqq x_{0}+\int_{t_{0}}^{t}[I(s, \lambda(s))+D(s, \Lambda(s))] d s$, and when we combine this result with the corresponding inequality that is obtained by applying Fatou's Lemma to the definition of $\Lambda(t)$, we see that

$$
\begin{aligned}
x_{0}+\int_{t_{0}}^{t}[I(s, \lambda(s)) & +D(s, \Lambda(s))] d s \\
& \leqq \lambda(t) \leqq \Lambda(t) \leqq x_{0}+\int_{t_{0}}^{t}[I(s, \Lambda(s))+D(s, \lambda(s))] d s .
\end{aligned}
$$

Now we abbreviate the expressions at the left and right ends of this set of inequalities by $\omega(t)$ and $\Omega(t)$, and we obtain the inequalities

$$
\omega(t) \leqq \lambda(t) \leqq \Lambda(t) \leqq \Omega(t) .
$$

From the definitions of $\omega$ and $\Omega$, we have $\omega^{\prime}(t)=I(t, \lambda(t))+D(t, \Lambda(t))$ and $\Omega^{\prime}(t)=I(t, \Lambda(t))+D(t, \lambda(t))$. In these equations we replace $\lambda(t)$ with $\omega(t)$ and $\Lambda(t)$ with $\Omega(t)$, and so, because of (8) and the monotonicity of $I$ and $D$, we obtain (4). Thus if we assume that the solution of (3) is unique, our lemma tells us that $\omega(t)=\Omega(t)$, and hence $\lambda(t)=\Lambda(t)$. We summarize these results as a theorem.

TheOREM. If the differential problem (3) has a unique solution, then (1) has a unique solution, and a Picard sequence defined by an initial function $\xi_{0}$ and the recursion formula (2) converges to this solution.

We may remark that if $f(t, x)$ is increasing in its second argument, then we can take $I=f$ and $D=0$, and so (3) is equivalent to (1). In this case, then, the uniqueness of the solution of (1) does imply the convergence of the Picard sequence. Therefore it is not surprising that examples that show that uniqueness of the solution of (1) does not imply convergence of the Picard sequence rely on functions that are decreasing in their second argument [1, p. 53].

Not only does the uniquenss of the solution of (3) guarantee the convergence of a sequence of successive approximations to the solution of (1), but it also guarantees that a sequence of pairs $\left(\eta_{n}(t), \zeta_{n}(t)\right)$ which we obtain by selecting an initial pair $\left(\eta_{0}(t), \zeta_{0}(t)\right)$ and applying the recursion formulas

$$
\begin{aligned}
& \eta_{n+1}(t)=x_{0}+\int_{t_{0}}^{t}\left[I\left(s, \eta_{n}(s)\right)+D\left(s, \zeta_{n}(s)\right)\right] d s, \\
& \zeta_{n+1}(t)=x_{0}+\int_{t_{0}}^{t}\left[I\left(s, \zeta_{n}(s)\right)+D\left(s, \eta_{n}(s)\right)\right] d s
\end{aligned}
$$

also converges (to the solution of (3), of course). This statement fol- 
lows from our lemma by much the same sort of argument that produced our basic theorem.

\section{REFERENCES}

1. E. A. Coddington and N. Levinson, Theory of ordinary differential equations, McGraw-Hill, New York, 1955.

2. L. M. Graves, The theory of functions of real variables, 2nd ed., McGraw-Hill, New York, 1956.

3. J. LaSalle, Uniqueness theorems and successive approximations, Ann. of Math. 50 (1949), 722-730.

Harpur College

\section{UNCOUNTABLY MANY NONISOMORPHIC NILPOTENT LIE ALGEBRAS ${ }^{1}$}

\section{CHONG-YUN CHAO}

Throughout this note, $L$ denotes a Lie algebra over the real number field $R$. We shall define $L^{i}$ and $L_{i}$ inductively. $L=L^{0}=L_{0}, L^{i}$ $=\left[L^{i-1}, L^{i-1}\right]$, and $L_{i}=\left[L, L_{i-1}\right]$ for all integers $i \geqq 1$. Thus, $L^{i}$ is the space of all finite sums $\sum[x, y], x, y \in L^{i-1}$. Similarly, $L_{i}$ is the space of all finite sums $\sum[x, y], x \in L$ and $y \in L_{i-1}$. If $L^{r}=0$ and $L^{r-1} \neq 0$, $L$ is said to be solvable of index $r$. If $L_{t}=0$ and $L_{t-1} \neq 0, L$ is said to be nilpotent of length $t$.

Definition. Let $F$ be a subfield of $R$. A Lie algebra $L$ over $R$ is said to be an $F$-algebra if its structure constants with respect to some basis of $L$ lie in $F$.

Malcev [1] showed that for each integer $n \geqq 16$ there is a nilpotent Lie algebra of length 2 and dimension $n$ which is not a rational algebra. The purpose of this note is to prove the following theorem which contains an improvement of Malcev's result:

THEOREM. There exist uncountably many nonisomorphic nilpotent Lie algebras of length 2 for any given dimension $N \geqq 10$.

Following from the theorem we can easily show:

Corollary 1. There exist uncountably many solvable not nilpotent Lie algebras of index 3 for any given dimension $M \geqq 11$.

Received by the editors November 13, 1961.

1 This is a portion of my thesis submitted to the University of Michigan. I am deeply grateful to Professor H. Samelson for his guidance and assistance. This work was supported by the contract AF 49(648)-104 and Lotta B. Backus scholarship. 